OLIVEIRA, RJP; GATIBONI, LC; VALICHESKI, RR; MIQUELLUTI, DJ; BRUNETTO, G. 2016. Calibração da adubação fosfatada e potássica para beterraba na região do Vale do Itajaí. Horticultura Brasileira 34: 210-215. DOI - http://dx.doi.org/10.1590/S0102-053620160000200010

\title{
Calibração da adubação fosfatada e potássica para beterraba na região do Vale do Itajaí
}

\author{
Robinson JP Oliveira' ${ }^{1}$; Luciano C Gatiboni ${ }^{2}$; Romano R Valicheski ${ }^{1}$; David J Miquelluti²; Gustavo \\ Brunetto $^{3}$ \\ ${ }^{1}$ Instituto Federal Catarinense (IFC), Rio do Sul-SC, Brasil; robijpo34@hotmail.com; romano.valicheski@ifc.edu.br; ${ }^{2}$ Universidade do \\ Estado de Santa Catarina (UESC), Departamento de Solos e Recursos Naturais, Lages-SC, Brasil; lgatiboni@gmail.com; a2djm@cav. \\ udesc.br; ${ }^{3}$ Universidade Federal de Santa Maria (UFSM), Centro de Ciências Rurais, Santa Maria-RS, Brasil; brunetto.gustavo@gmail. \\ com
}

\section{RESUMO}

Estudos sobre adubação na cultura de beterraba ainda são necessários em algumas regiões de Santa Catarina. Assim, o objetivo foi avaliar a resposta da cultura da beterraba a doses de fósforo $(\mathrm{P})$ e potássio $(\mathrm{K})$ em Cambissolo Háplico, que é o tipo de solo predominante em $70 \%$ da região do Alto Vale do Itajaí. O experimento foi conduzido em Rio do Sul-SC em dois cultivos, sendo o primeiro cultivo instalado em blocos inteiramente casualizados no esquema fatorial $4 \times 4\left(0,200,400\right.$ e $\left.600 \mathrm{~kg} / \mathrm{ha} \mathrm{de} \mathrm{P}_{2} \mathrm{O}_{5}\right)$ e $(0,150,300$ e 450 $\mathrm{kg} /$ ha de $\mathrm{K}_{2} \mathrm{O}$ ) com 3 repetições. No segundo cultivo, foi avaliada a reposição de $\mathrm{P} \mathrm{e} \mathrm{K}$, onde cada parcela do primeiro cultivo foi dividida em três, sendo aplicados 0, 33 e $66 \%$ das doses iniciais dos nutrientes. Após 60 dias do transplantio foram avaliados diâmetro de raízes, e massa fresca e seca de folhas e raízes. No primeiro cultivo, houve efeito significativo $(\alpha=5 \%)$ para o fator P para massa seca e fresca de raiz e diâmetro de raiz e não houve efeito do $\mathrm{K}$, mas a exportação foi elevada, atingindo $172,27 \mathrm{~kg} / \mathrm{ha}$ de $\mathrm{K}$ com a dose $418 \mathrm{~kg} / \mathrm{ha}$ de $\mathrm{K}_{2} \mathrm{O}$. A máxima produção ocorreu com $379 \mathrm{~kg} / \mathrm{ha}$ de $\mathrm{P}_{2} \mathrm{O}_{5}$, sendo que o exportado foi de $21,30 \mathrm{~kg} / \mathrm{ha}$ de $\mathrm{P}$ na massa seca de raízes e folhas. No segundo cultivo, a beterraba respondeu à aplicação de $\mathrm{P}$ e $\mathrm{K}$, sendo a máxima produtividade obtida com as maiores doses de $\mathrm{P}$ e K (450 kg/ha de $\mathrm{K}_{2} \mathrm{O}$ e $600 \mathrm{~kg} / \mathrm{ha}$ de $\mathrm{P}_{2} \mathrm{O}_{5}$ ) e de reposição (300 $\mathrm{kg} / \mathrm{ha}$ de $\mathrm{K}_{2} \mathrm{O}$ e $400 \mathrm{~kg} / \mathrm{ha}$ de $\mathrm{P}_{2} \mathrm{O}_{5}$ ). Conclui-se que a beterraba é uma cultura muito exigente em fertilização com $\mathrm{P}$ e K, respondendo a doses maiores do que aquelas recomendadas pelos manuais de adubação utilizados na região.

Palavras-chave: Beta vulgaris, nutrição mineral, crescimento, produção, solo.

\begin{abstract}
Calibration of phosphorus and potassium fertilization for beet in Vale do Itajaí region

Studies on fertilization for beet are still needed in some regions of Santa Catarina State, Brazil. The objective of this work was to evaluate the response of beet to phosphorus $(\mathrm{P})$ and potassium $(\mathrm{K})$ in an Haplic Cambisol, which is the predominant soil type in $70 \%$ of the Alto Vale do Itajaí region. The experiment was carried out in Rio do Sul, Santa Catarina, in two crops. The first cultivation was in randomized blocks in a factorial $4 \times 4$ design $(0,200,400$ and $600 \mathrm{~kg} /$ ha $\left.\mathrm{P}_{2} \mathrm{O}_{5}\right)$ and $\left(0,150,300\right.$ and $\left.450 \mathrm{~kg} / \mathrm{ha} \mathrm{K}_{2} \mathrm{O}\right)$, with 3 replications. On the second crop, the rates of $\mathrm{P}$ and $\mathrm{K}$ for replacement were assessed, each original plot being splitted into three and applied 0, 33 and $66 \%$ of the initial rate of $\mathrm{P}$ and $\mathrm{K}$. At 60 days after transplanting, diameter of roots and fresh and dry weight of leaves and roots were evaluated. In the first crop, a significant effect $(\alpha=5 \%)$ was observed for the $\mathrm{P}$ factor on dry and fresh weight of roots as well as on root diameter. On the other hand, no effect of $\mathrm{K}$ was noticed, but $\mathrm{K}$ exportation by plants was high, reaching $172.27 \mathrm{~kg} / \mathrm{ha} \mathrm{K}$ with the dose $418 \mathrm{~kg} /$ ha $\mathrm{K}_{2} \mathrm{O}$. The maximum production took place with $379 \mathrm{~kg} / \mathrm{ha} \mathrm{P}_{2} \mathrm{O}_{5}$, and exportation was $21.30 \mathrm{~kg} / \mathrm{ha} \mathrm{P}$ on dry mass of roots and leaves. In the second crop, plants responded to application of $\mathrm{P}$ and $\mathrm{K}$, and the maximum yield was obtained with higher initial rates $(450 \mathrm{~kg} /$ ha $\mathrm{K}_{2} \mathrm{O}$ and $\left.600 \mathrm{~kg} / \mathrm{ha} \mathrm{P}_{2} \mathrm{O}_{5}\right)$ and replacement rates $\left(300 \mathrm{~kg} / \mathrm{ha} \mathrm{K}_{2} \mathrm{O}\right.$ and $400 \mathrm{~kg} / \mathrm{ha} \mathrm{P}_{2} \mathrm{O}_{5}$ ). Beet is a crop highly demanding on nutrients, with needs of $\mathrm{P}$ and $\mathrm{K}$ higher than those recommended by local fertilization tutorials.
\end{abstract}

Keywords: Beta vulgaris, mineral nutrition, growth, yield, soil.

(Recebido para publicação em 22 de janeiro de 2015; aceito em 20 de janeiro de 2016) (Received on January 22, 2015; accepted on January 20, 2016)

\begin{abstract}
A beterraba (Beta vulgaris) é uma hortaliça que vem ganhando espaço e importância econômica no Brasil, sendo uma das dez principais olerícolas cultivadas (Sediyama et al., 2011). Em Santa Catarina-SC, o volume médio comercializado dessa hortaliça foi de 3.600 t/ano, gerando um volume mo-
\end{abstract}

netário equivalente a $\mathrm{R} \$$ 4,00 milhões, de acordo com os dados da CEASA-SC (2007). Na região do Alto Vale do Itajaí, o cultivo da beterraba vem crescendo nos últimos anos e ocupa uma área de 773,1 ha, envolvendo diretamente 296 famílias (EPAGRI, 2010). Esse crescimento da cultura é devido à necessida- de de diversificação da produção nas propriedades familiares após o cultivo da cebola, que é a principal cultura da região (Duarte et al., 2012).

No Brasil, são escassos os estudos relacionados à adubação, nutrição mineral de beterraba e quantidade de nutrientes exportados (Grangeiro et al., 2007). 
Quanto à adubação, Filgueira (2012) apresenta uma sugestão para locais onde não existam ainda dados regionais. Por outro lado, há vários trabalhos sobre beterraba açucareira em outros países, os quais mostram a dependência da cultura à adubação NPK (Marinković et al., 2010; Turk, 2010).

A adubação com fósforo $(\mathrm{P})$ nas hortaliças favorece o desenvolvimento do sistema radicular e reflete no aumento da produtividade dos produtos colhidos (Avalhaes et al., 2009). Embora sua exigência seja baixa, é fundamental seu fornecimento nos solos tropicais devido aos baixos teores disponíveis às plantas.

O potássio (K), por sua vez, é absorvido em maior quantidade pelas raízes da beterraba (Campo et al., 2000) com importante função na translocação e armazenamento de fotoassimilados e na manutenção de água pela planta. É conhecido como o nutriente que promove aumento da qualidade devido aos seus efeitos no tamanho, forma, cor, sabor e resistência dos produtos hortícolas ao armazenamento (Chitarra \& Chitarra, 2005).

A necessidade do uso de uma adubação equilibrada é fundamental para o fornecimento correto da quantidade de nutrientes exigido pela cultura. Entretanto, a aplicação de nutrientes na região do Alto Vale do Itajaí é feita, atualmente, conforme o Manual de Adubação e Calagem para o Estado de Santa Catarina e Rio Grande do Sul (CQFS-RS/SC, 2004), cujas doses dos nutrientes são genéricas para as culturas da beterraba e cenoura ou conforme o Manual de Olericultura (Filgueira, 2012). Aliado a isso, há grande disparidade entre as doses recomendadas.

Para uma adubação adequada da cultura, é necessário relacionar o teor disponível no solo, quantidade a ser aplicada e o rendimento da cultura. A obtenção de nível crítico do $\mathrm{P}$ e $\mathrm{K}$ no solo indicará qual o teor no solo a partir do qual a probabilidade de resposta é pequena ou nula, podendo prever a resposta da cultura da beterraba à adubação, além da quantidade de fertilizante a ser utilizada caso o teor do nutriente esteja abaixo do nível crítico (CQFS-RS/SC, 2004).

Assim, o presente trabalho obje- tivou avaliar a resposta da cultura da beterraba a doses de fósforo e potássio em Cambissolo Háplico, que é o tipo de solo predominante em $70 \%$ da região do Alto Vale do Itajaí.

\section{MATERIAL E MÉTODOS}

O trabalho foi conduzido na área experimental do Instituto Federal Catarinense em Rio do Sul-SC com a cultivar de beterraba Early Wonder em dois cultivos. No primeiro cultivo (06/07/2010 a 03/09/2010), o volume de precipitação, temperaturas mínimas e máximas foram de $337,5 \mathrm{~mm}, 9,05^{\circ} \mathrm{C}$ e $20,18^{\circ} \mathrm{C}$, respectivamente. No segundo cultivo (06/12/2010 a 04/02/2011), o volume de precipitação foi de $469 \mathrm{~mm}$ com temperaturas entre $17,44^{\circ} \mathrm{C}$ e $29,91^{\circ} \mathrm{C}$.

$\mathrm{O}$ solo da área experimental foi um Cambissolo Háplico (argila= 240 g/kg) com os seguintes atributos químicos: $\mathrm{pH}$ (água) $=5,2 ; \mathrm{P}$ e K $=2,5$ e $30 \mathrm{mg} / \mathrm{dm}^{3}$; $\mathrm{Ca}, \mathrm{Mg}, \mathrm{Al}$ e CTC: 4,$2 ; 2,2 ; 0,8$ e 12,6 $\mathrm{cmol} / \mathrm{dm}^{3}$, respectivamente; saturação por bases $(\mathrm{V} \%)=51,3 \%$. A correção do solo foi realizada, conforme recomendação da CQFS-RS/SC (2004), para elevar o pH do solo a 6,0, sendo utilizado 5,2 t/ ha de calcário calcítico $(\mathrm{CaO}=46,44 \%$, $\mathrm{MgO}=5,20 \%$ e $\mathrm{PRNT}=92 \%$ ).

As mudas foram produzidas em bandejas de poliestireno expandido em casa de vegetação, sendo feito o transplantio 30 dias após a semeadura para canteiros com $1 \mathrm{~m}$ de largura. As plantas foram conduzidas no espaçamento $8 \mathrm{~cm}$ entre plantas e $25 \mathrm{~cm}$ entre linhas (Filgueira, 2012). Cada parcela possuía $80 \mathrm{~cm}$ de comprimento e $30 \mathrm{~cm}$ de bordadura. Antes do transplantio das mudas foram aplicados $30 \mathrm{~kg} / \mathrm{ha}$ de N, 1, $0 \mathrm{~kg} / \mathrm{ha} \mathrm{de} \mathrm{B}$ e $2,0 \mathrm{~kg} / \mathrm{ha}$ de $\mathrm{Zn}$. O restante do N (80 $\mathrm{kg} / \mathrm{ha}$ ) foi aplicado em cobertura aos 20 e 40 dias após o transplantio. Esse procedimento foi feito nos dois cultivos.

No $1^{\circ}$ cultivo foi avaliada a curva de resposta a diferentes doses de $\mathrm{P}$ e $\mathrm{K}$. O experimento foi instalado em blocos ao acaso, no esquema fatorial $4 \times 4$, sendo avaliadas doses de fósforo $(0,200,400$ e $600 \mathrm{~kg} / \mathrm{ha})$ e de potássio $(0,150,300$ e $450 \mathrm{~kg} / \mathrm{ha}$ ), totalizando 16 tratamentos com três repetições. As doses foram definidas de modo a abranger as reco- mendações de $\mathrm{P}$ e K, segundo Filgueira (2012) e CQFS-RS/SC (2004), conforme análise química inicial do solo. Em cada parcela experimental, foram cultivadas 40 plantas das quais, foram coletadas 4 plantas para avaliação.

No $2^{\circ}$ cultivo foi testada a curva de resposta das plantas à reposição de $\mathrm{P} \mathrm{e}$ $\mathrm{K}$ após a primeira adubação.

Cada parcela do $1^{\circ}$ cultivo foi subdividida em 3 partes, sendo que as parcelas do $2^{\circ}$ cultivo apresentaram $26 \mathrm{~cm}$ de comprimento, onde foram aplicadas 0,33 ou $66 \%$ da dose de fertilizante anterior. Em cada parcela experimental foram cultivadas 16 plantas, sendo coletadas quatro para avaliação. As aplicações de nitrogênio, boro e zinco foram realizadas na mesma época e doses aplicadas no primeiro cultivo.

Em ambos os cultivos, a coleta das plantas foi feita aos 60 dias após o transplante, determinando-se o diâmetro de raiz e massa fresca e seca de folhas e raízes e os teores de $\mathrm{P}$ e $\mathrm{K}$ no material vegetal seco e moído (Tedesco, 1995).

Os dados avaliados no primeiro cultivo foram submetidos à análise de variância e análise de regressão com auxílio do programa SAS (Statistical Analysis System, 2003). Para o segundo cultivo, as curvas de resposta às doses de $\mathrm{P}$ foram feitas, considerando as aplicações nos dois cultivos, para cada dose de $\mathrm{K}$ aplicada no primeiro cultivo. A normalidade e a homogeneidade de variâncias dos resíduos foram testadas pelos testes de Shapiro-Wilk e de Hartley (F máximo). Para as equações de ajuste da dose de $\mathrm{P},(\mathrm{X} 1)$ refere-se à dose de fósforo aplicada no primeiro cultivo e (X2) à dose de fósforo aplicada no segundo cultivo.

\section{RESULTADOS E DISCUSSÃO}

\section{Primeiro cultivo}

A adubação com fósforo influenciou a massa seca de raiz (MSR), massa fresca de raiz (MFR) e diâmetro de raiz (DR). No entanto, não houve resposta às doses de $\mathrm{K}$ aplicadas, indicando que os teores disponíveis no solo foram suficientes para atender a demanda das plantas (Tabela 1).

A produção máxima de massa fresca 
de raiz foi de 29,17 t/ha (equivalente a produto beneficiado), obtida com a aplicação de $470 \mathrm{~kg} / \mathrm{ha}$ de P. Esse valor está dentro dos valores médios preconizados para a cultura, sendo a produtividade da beterraba beneficiada (raízes) extremamente variável, situando-se entre 20 e 35 t/ha (Resende \& Cordeiro, 2007; Filgueira, 2012).

Os valores máximos de massa seca de raiz (39,3 g/planta) e diâmetro de raiz $(3,91 \mathrm{~cm})$ foram obtidos com 385 e 375 $\mathrm{kg} / \mathrm{ha}$ de P (Figura 1), respectivamente. Esses resultados de massa fresca e seca de raiz e diâmetro mostram que as doses de $\mathrm{P}$ máximas nesse trabalho foram superiores àquelas recomendadas por Filgueira (2012), cujos valores são entre 200 a $350 \mathrm{~kg} / \mathrm{ha}$ de $\mathrm{P}_{2} \mathrm{O}_{5}$ e a proposta para solos com características similares (classe textural 3; teor muito baixo de $\mathrm{P}=2,5 \mathrm{mg} / \mathrm{dm}^{3}$ ) pelo CQFS-RS/SC (2004), que sugere $240 \mathrm{~kg} / \mathrm{ha}$ de $\mathrm{P}_{2} \mathrm{O}_{5}$.

$\mathrm{O}$ teor máximo de $\mathrm{P}$ na folha $(7,82$ $\mathrm{g} / \mathrm{kg}$ ) foi obtido com $333 \mathrm{~kg} / \mathrm{ha}$ de $\mathrm{P}$, equivalente à exportação de $7,82 \mathrm{~kg} / \mathrm{ha}$ para uma lavoura com 500.000 plantas/ ha (Figura 2). Isso foi superior ao obtido por Grangeiro et al. (2007), que obtiveram $4,16 \mathrm{~kg} / \mathrm{ha}$ de $\mathrm{P}$ acumulado em folhas para uma população de 330.000 plantas/ha, equivalente a $6,30 \mathrm{~kg} / \mathrm{ha}$ para uma população de 500.000 plantas/ha.

Quanto ao teor de P nas raízes, houve incremento com o aumento das doses aplicadas, obtendo-se valor máximo com a aplicação de $409 \mathrm{~kg} / \mathrm{ha}$ de P. Para essa dose, a exportação total de $\mathrm{P}$ nas raízes foi de $13,55 \mathrm{~kg} / \mathrm{ha}$ para 500.000 plantas/ha, resultados superiores aos obtidos por Grangeiro et al. (2007) para 330.000 plantas $(6,06 \mathrm{~kg} / \mathrm{ha}$ de $\mathrm{P})$.

A máxima exportação de $P$ pela planta de beterraba ocorreu com a dose de $379 \mathrm{~kg} / \mathrm{ha}$. Considerando uma lavoura com 500.000 plantas/ha (recomendação para a região), o total de $\mathrm{P}$ acumulado pelas plantas foi de $21,30 \mathrm{~kg} / \mathrm{ha}$. Haag \& Minami (1987), ao trabalharem com a cultivar Early Wonder, mostraram que a extração de $\mathrm{P}$ foi contínua dos 40 dias até a colheita ( 80 dias), sendo máxima absorção aos 60 dias de idade. Considerando a exportação de P pela planta (raiz e parte aérea), os valores obtidos para $\mathrm{P}$ foram superiores aos encontrados por
Grangeiro et al. (2007) onde os mesmos destacaram o acúmulo de $15,48 \mathrm{~kg} / \mathrm{ha}$ de Pna planta. Em relação à extração de $\mathrm{P}$, as raízes apresentaram maior contribuição $(63,29 \%)$ comparado com a parte aérea $(36,71 \%)$. Esses dados corroboraram com Magro (2012), que relatou a extração de $63 \%$ do $\mathrm{P}$ absorvido pelas raízes e de $37 \%$ para a parte aérea em plantas ao final do ciclo.

Embora não tenha ocorrido resposta significativa para as variáveis biométricas (massa seca e fresca de folha e raiz e diâmetro de raiz), as doses de $\mathrm{K}$ influenciaram a exportação desse nutriente pelas raízes. A adubação com potássio proporcionou maior exportação de $\mathrm{K}$ comparado à testemunha (Figura 3), indicando o fenômeno de "consumo de luxo", onde a alta disponibilidade de um nutriente faz com que a sua absorção e exportação pelas culturas seja grande, mesmo sem resposta em produtividade com o uso do nutriente. A dose $320 \mathrm{~kg} /$ ha de $\mathrm{K}$ proporcionou maior acúmulo de $\mathrm{K}$ na raiz $(3,55 \mathrm{~g} / \mathrm{kg})$, o que equivale a exportação de 103,72 kg/ha de K. Na planta, houve também efeito da adubação de potássio, sendo que a dose 418 $\mathrm{kg} / \mathrm{ha}$ de $\mathrm{K}$ proporcionou um acúmulo máximo de $\mathrm{K}$ de $172,27 \mathrm{~kg} / \mathrm{ha}$ na planta. Isso concorda com Grangeiro et al. (2007) que relataram exportação total de $179,3 \mathrm{~kg} / \mathrm{ha}$ para a mesma cultivar aos 60 dias após o transplantio das mudas. Embora não tenha ocorrido resposta à adubação potássica no presente experimento, a quantidade de $\mathrm{K}$ exportada

Tabela 1. Valores de F e nível de significância para as diversas fontes de variação e coeficiente de variação para as variáveis biométricas avaliadas; MSR= massa seca de raiz; $\mathrm{MSF}=$ massa seca de folha; $\mathrm{MFR}=$ massa fresca de raiz; $\mathrm{MFF}=$ massa fresca de folha; $\mathrm{DR}=$ diâmetro de raiz ( $\mathrm{F}$ values and significance level for the different sources of variation and coefficient of variation for biometric variables evaluated; $\mathrm{MSR}=$ dry mass of roots; $\mathrm{MSF}=$ leaf dry mass; $\mathrm{MFR}=$ fresh weight of root; $\mathrm{MFF}=$ fresh weight of leaf, $\mathrm{DR}=$ diameter of root). Rio do Sul, IFC, 2013.

\begin{tabular}{llllll}
\hline FV & MSR & MSF & MFR & MFF & DR \\
\hline Bloco & $3,84^{*}$ & $0,73^{\text {ns }}$ & $8,49^{* *}$ & $6,63^{* *}$ & $5,73^{* *}$ \\
Fósforo & $5,56^{* *}$ & $0,48^{\text {ns }}$ & $4,75^{* *}$ & $1,97^{\text {ns }}$ & $3,64^{*}$ \\
Potássio & $0,89^{\text {ns }}$ & $0,81^{\text {ns }}$ & $2,24^{\text {ns }}$ & $2,03^{\text {ns }}$ & $1,68^{\text {ns }}$ \\
P x K & $0,94^{\text {ns }}$ & $0,57^{\text {ns }}$ & $1,00^{\text {ns }}$ & $2,03^{\text {ns }}$ & $0,92^{\text {ns }}$ \\
\hline CV $(\%)$ & 24,6 & 17,3 & 23,6 & 25,9 & 13,4 \\
\hline
\end{tabular}

ns= não significativo (non significant), *=significativo (significant), **=altamente significativo (highly significant) $(\alpha=5 \%)$.

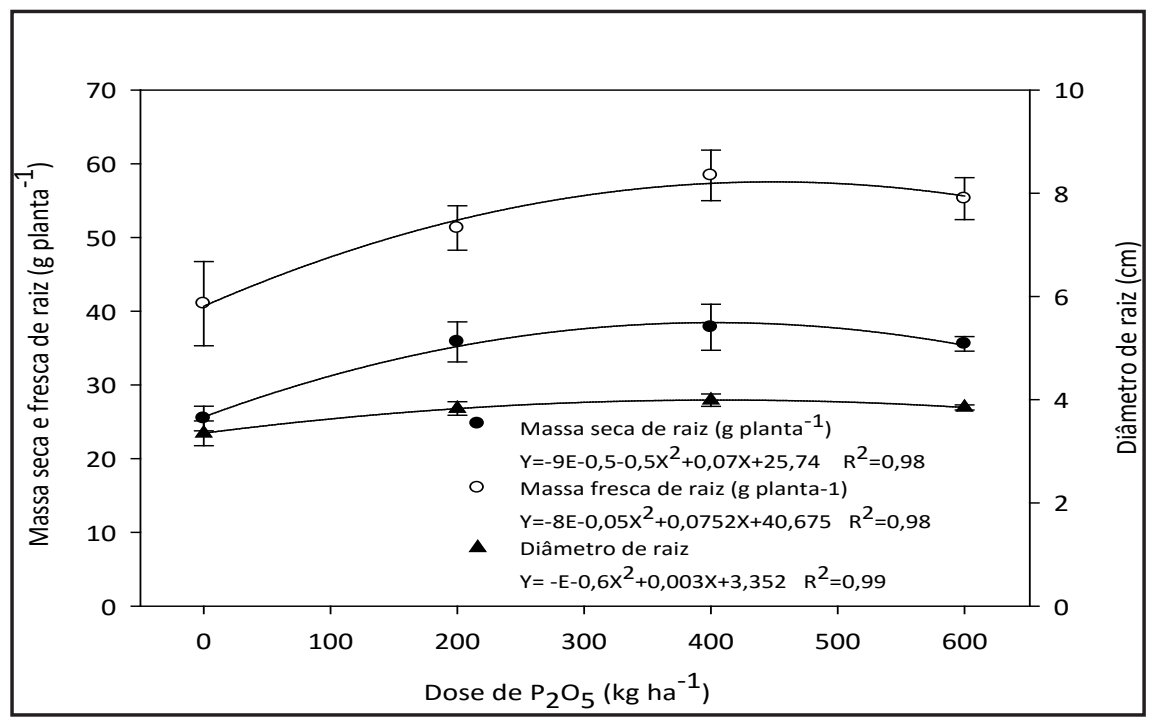

Figura 1. Massa seca, massa fresca e diâmetro de raiz, em função das doses de fósforo aplicadas (dry weight, fresh weight and diameter of roots, depending on phosphorus doses). Rio do Sul, IFC, 2013. 


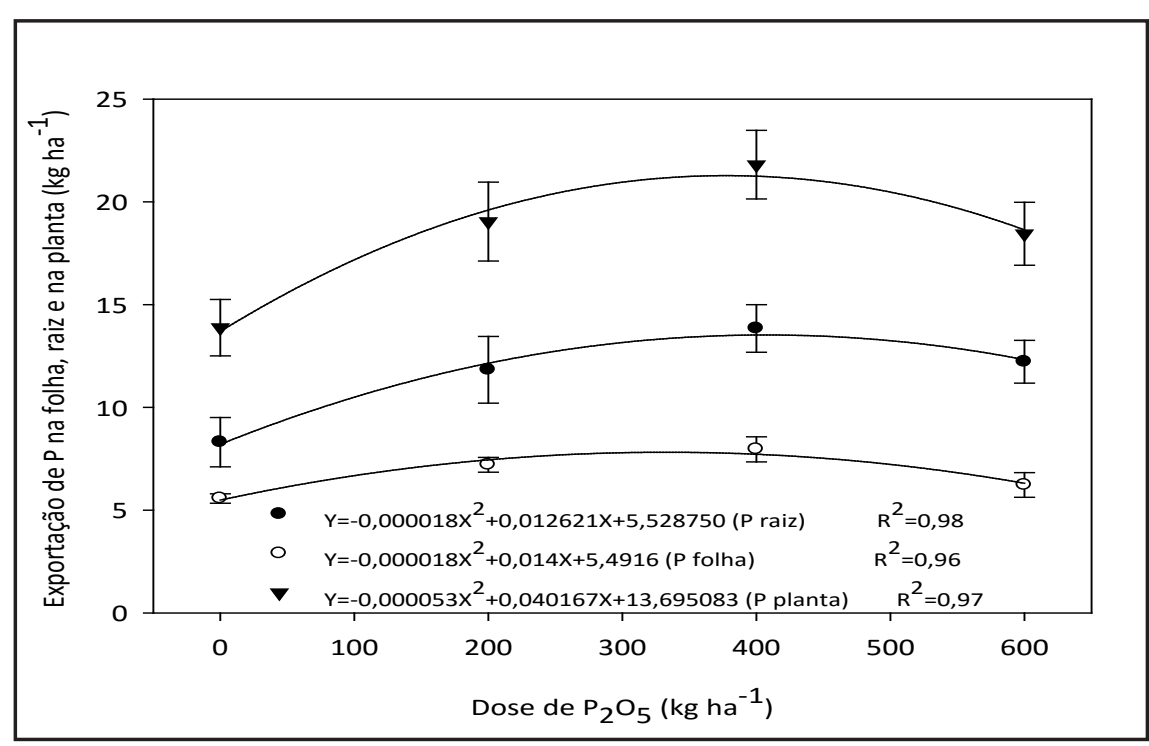

Figura 2. Exportação de fósforo na folha, raiz e na planta em função da dose de $\mathrm{P}_{2} \mathrm{O}_{5}(\mathrm{~kg} /$ ha) aplicada no solo \{exportation of phosphorus in the leaf, root and plant depending on the dose of $\mathrm{P}_{2} \mathrm{O}_{5}(\mathrm{~kg} / \mathrm{ha})$ applied to the soil\}. Rio do Sul, IFC, 2013.

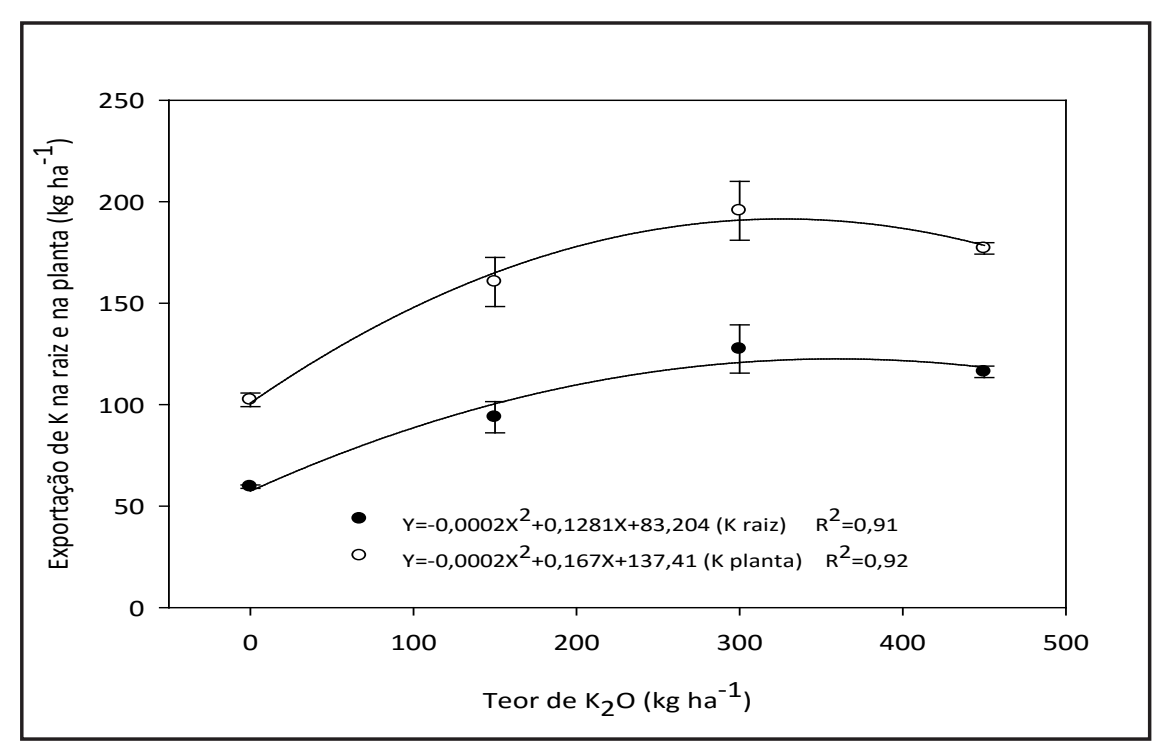

Figura 3. Exportação de potássio na raiz e na planta em função da dose de $\mathrm{K}_{2} \mathrm{O}$ (kg/ha) aplicados no solo (exportation of potassium on root and plant depending on dose of $\mathrm{K}_{2} \mathrm{O}$ $(\mathrm{kg} / \mathrm{ha})$ applied to the soil\}. Rio do Sul, IFC, 2013.

via colheita é um fator importante para o ajuste da adubação dos cultivos subsequentes. O elevado acúmulo de $\mathrm{K}$ na parte aérea e raiz da beterraba mostram sua importância para plantas armazenadoras de reserva em órgão subterrâneos, que faz dele o nutriente mais extraído pela planta para translocação de açúcares e síntese de amido e requerido para a obtenção de produções elevadas (Alves et al., 2008).

Quanto à participação da parte aérea drico. Isso pode ter limitado a absorção de $\mathrm{K}$ pelas plantas, o que levou a uma redistribuição do K que estava nas folhas para o órgão em crescimento, no caso a raiz, justificando assim, a maior proporção de $\mathrm{K}$ nas raízes em relação à parte aérea. Para a extração de K pelas folhas, não houve resposta às doses de $\mathrm{K}$. Segundo Khatounian (2001), o K é um elemento ativo em forma livre, sendo prontamente liberado para o solo, principalmente onde a CTC é menor. Como nas folhas a demanda é menor, quando comparado com a raiz, acredita-se que a quantidade fornecida pelo solo atende às necessidades da parte aérea da planta não diferindo estatisticamente entre si em razão da dose de K aplicada no solo.

Com base no Manual de adubação e de calagem (CQFS-RS/SC, 2004), o teor crítico para o K no solo (com CTC de $12,6 \mathrm{mg} / \mathrm{kg}$ ) é de $60 \mathrm{mg} / \mathrm{kg}$, e no solo trabalhado o teor original de K é de $30 \mathrm{mg} /$ $\mathrm{kg}$ (análise de solo inicial), indicando, a princípio, a necessidade de adubação potássica. Contudo, o rendimento relativo da beterraba em função da disponibilidade de K existente no solo apontou um nível crítico menor que o preconizado $(47 \mathrm{mg} / \mathrm{kg})$. Esse menor valor crítico para $\mathrm{o} \mathrm{K}$ e a ausência de resposta às doses de $\mathrm{K}$ nas variáveis biométricas avaliadas pode estar relacionado ao fato do experimento ser desenvolvido em um Cambissolo Háplico com reservas de $\mathrm{K}$ não trocável. Segundo Almeida et al. (1997), os Cambissolos da Região de Rio do Sul apresentam a mineralogia da fração argila composta de caulinita como argilomineral dominante, além de mica (ou ilita), argilominerais 2:1 com ou sem polímeros de hidroxi-Al nas entrecamadas, interestratificados e quartzo. Esses argilominerais do tipo 2:1 devido os fatores do intemperismo agir de modo gradual, podem estar liberando parte do K que a beterraba necessita para o seu desenvolvimento, contribuindo para o abaixamento do nível crítico da cultura. Comportamento similar foi encontrado por Kaminski et al. (2010) em Argissolos da região de Santa Maria-RS, onde a presença de minerais 2:1 contendo $\mathrm{K}$ reduziram o nível crítico para $51 \mathrm{mg} / \mathrm{kg}$ (abaixo de $60 \mathrm{mg} / \mathrm{kg}$ ) para um experimento de longa duração com culturas de grãos. 
Tabela 2. Equações de ajuste de doses de $\mathrm{P}$ no $1^{\circ}$ cultivo (X1) e no segundo cultivo (X2) em cada nível de potássio aplicado no $1^{\circ}$ cultivo para as variáveis de produção de massa fresca e seca, sendo apresentados os dados apenas para os parâmetros e doses de K onde foi observado efeito significativo \{adjustment equations for $\mathrm{P}$ doses on the $1^{\text {st }}$ cultivation for the variables production of fresh and dry mass, data being presented only for the parameters and dosis of $\mathrm{K}$ with a significant effect\}. Rio do Sul, IFC, 2013.

\begin{tabular}{|c|c|c|}
\hline Dose de $\mathrm{K}_{2} \mathrm{O}$ (kg/ha) & Equação & Valor de $\mathbf{R}^{2}$ \\
\hline & Massa fresca de folha & \\
\hline \multirow[t]{2}{*}{$\mathrm{K} 1=150$} & $\mathrm{Y}=13,8933+0,0227 \mathrm{X}_{1}$ & $\mathrm{R}^{2}=0,16$ \\
\hline & Massa fresca de raiz & \\
\hline \multirow[t]{2}{*}{$\mathrm{K} 1=450$} & $Y=45,5771+0,1003 X_{1}+0,2558 X_{2}$ & $\mathrm{R}^{2}=0,62$ \\
\hline & Massa seca de raiz & \\
\hline \multirow[t]{2}{*}{$\mathrm{K} 1=450$} & $\mathrm{Y}=4,9894+0,0204 \mathrm{X}_{1}-0,00001 \mathrm{X}_{1}^{2}+0,0126 \mathrm{X}_{2}$ & $\mathrm{R}^{2}=0,59$ \\
\hline & Diâmetro de raiz & \\
\hline $\mathrm{K} 1=150$ & $\mathrm{Y}=1,4867+0,0027 \mathrm{X}_{1}-0,00000077 \mathrm{X}_{1}^{2}+0,0100 \mathrm{X}_{2}-0,0000 \mathrm{X}_{2}^{2}$ & $\mathrm{R}^{2}=0,65$ \\
\hline
\end{tabular}

Nas equações, $\mathrm{X}_{1}=\mathrm{P}$ no primeiro cultivo ( $\mathrm{P}$ on the first cultivation); $\mathrm{X}_{2}=\mathrm{P}$ no segundo cultivo ( $\mathrm{P}$ on the second cultivation).

Tabela 3. Equações de ajuste de doses de P no $1^{\circ}$ cultivo (X1) e no segundo cultivo (X2) em cada nível de potássio aplicado no $1^{\circ}$ cultivo para as variáveis de $\mathrm{P}$ e K no tecido vegetal, sendo apresentados os dados apenas para os parâmetros e doses de K onde foi observado efeito significativo $(\mathrm{P}<0,05)$ \{adjustment equations for $\mathrm{P}$ levels in the $1^{\text {st }}$ crop $(\mathrm{X} 1)$ and the second crop $(\mathrm{X} 2)$ at each level of potassium applied in the $1^{\text {st }}$ crop for the variables of $\mathrm{P}$ and $\mathrm{K}$ in plant tissue. Data are presented only for the parameters and doses where $\mathrm{K}$ had significant effect $(\mathrm{P}<0.05)\}$. Rio do Sul, IFC, 2013.

\begin{tabular}{|c|c|c|}
\hline Variáveis & $\mathrm{K}=0 \mathrm{~kg} / \mathrm{ha}$ de $\mathrm{K}_{2} \mathrm{O}$ & \\
\hline $\mathrm{K}$ na raiz & $Y=50,2234+0,0052 X_{1}+0,7321 X_{2}-0,0009 X_{1} X_{2}$ & $\mathrm{R}^{2}=0,49$ \\
\hline$P$ na raiz & $Y=7,2471-0,0046 X_{1}+0,0374 X_{2}$ & $\mathrm{R}^{2}=0,49$ \\
\hline \multicolumn{3}{|c|}{$\mathrm{K}=150 \mathrm{~kg} / \mathrm{ha}$ de $\mathrm{K}_{2} \mathrm{O}$} \\
\hline $\mathrm{K}$ na raiz & $Y=56,7125-0,0226 X_{1}+0,66814 X_{2}-0,0013 X_{2}^{2}$ & $\mathrm{R}^{2}=0,42$ \\
\hline $\mathrm{K}$ na folha & $Y=19,9391+0,0483 X_{1}+0,3094 X_{2}-0,0008 X_{2}^{2}$ & $\mathrm{R}^{2}=0,38$ \\
\hline $\mathrm{P}$ na raiz & $Y=8,2086+0,0226 X_{1}$ & $\mathrm{R}^{2}=0,22$ \\
\hline P na folha & $Y=6,9225+0,0543 X_{2}-0,00013 X_{2}^{2}$ & $\mathrm{R}^{2}=0,24$ \\
\hline \multicolumn{3}{|c|}{$\mathrm{K}=450 \mathrm{~kg} / \mathrm{ha}$ de $\mathrm{K}_{2} \mathrm{O}$} \\
\hline $\mathrm{K}$ na raiz & $Y=73,3632+0,2404 X_{2}$ & $\mathrm{R}^{2}=0,36$ \\
\hline $\mathrm{K}$ na folha & $Y=31,6094+0,1733 X_{2}$ & $\mathrm{R}^{2}=0,38$ \\
\hline$P$ na raiz & $\mathrm{Y}=9,0108+0,0402 \mathrm{X}_{2}$ & $\mathrm{R}^{2}=0,40$ \\
\hline P na folha & $Y=7,5722+0,0235 X_{2}$ & $\mathrm{R}^{2}=0,36$ \\
\hline
\end{tabular}

Nas equações, $\mathrm{X}_{1}=\mathrm{P}$ no primeiro cultivo ( $\mathrm{P}$ on the first cultivation); $\mathrm{X}_{2}=\mathrm{P}$ no segundo cultivo ( $\mathrm{P}$ on the second cultivation).

Em relação ao $\mathrm{P}$, o nível crítico estabelecido no Manual de adubação e de calagem (CQFS-RS/SC, 2004) para solos com $240 \mathrm{~g} / \mathrm{kg}$ de argila (classe textural 3), é de $12 \mathrm{mg} / \mathrm{kg}$ extraído pelo método Melich-1. O valor crítico obtido neste trabalho $(18,5 \mathrm{mg} / \mathrm{kg})$, que foi superior ao valor apresentado, justifica a resposta obtida às doses de $\mathrm{P}$ aplicadas acima do recomendado.

\section{Segundo cultivo}

O cultivo intensivo das hortaliças exige uma grande reposição de nutrientes em forma de adubação devido à elevada exportação. Dependendo da espécie cultivada, exige a aplicação de doses elevadas de fertilizantes (Raij et al., 1997) para a obtenção de altos rendimentos e boa qualidade. Em função da não resposta ao $\mathrm{K}$ no primeiro cultivo, a análise estatística para o $2^{\circ}$ cultivo foi realizada individualmente para cada nível de K. Assim, em cada nível, foi testada a resposta ao $\mathrm{P}$ (efeito residual do primeiro cultivo e efeito imediato das doses de reposição no segundo cultivo).

Quanto à massa fresca e seca de raiz (Tabela 2), o maior valor foi obtido para os tratamentos de plantio com dose máxima de fertilizante aplicada (450 $\mathrm{kg} /$ ha de $\mathrm{K}$ e $600 \mathrm{~kg} /$ ha de P) com dose de reposição de fertilizante em $66 \%$ da dose de plantio, onde a produtividade chegou a 25,88 t/ha. Isso mostra que, no segundo cultivo, a cultura respondeu até a maior dose de $\mathrm{P}$ e $\mathrm{K}$ aplicados, devido à sua alta exportação ocorridos no primeiro cultivo, necessitando altas doses de reposição. Já para $\mathrm{P}$ a resposta às doses de $\mathrm{P}$ foram ainda elevadas devido à adsorção do $\mathrm{P}$ residual ao solo (Novais \& Smyth, 1999).

Para diâmetro de raiz (Tabela 2), o maior valor foi obtido com $150 \mathrm{~kg} / \mathrm{ha}$ de $\mathrm{K}$ e $600 \mathrm{~kg} / \mathrm{ha}$ de $\mathrm{P}$ no $1^{\circ}$ cultivo e reposição em 33\% atingindo assim, 3,75 $\mathrm{cm}$ de diâmetro de raiz. Similarmente, para massa fresca de folha (Tabela 2) as 
doses foram as mesmas, independente da dose de reposição.

Não houve diferença quanto ao volume de $\mathrm{K}$ exportado pelas raízes (Tabela 3) em função da adubação de reposição para a dose $0 \mathrm{~kg} / \mathrm{ha}$ de $\mathrm{K}_{2} \mathrm{O}$, sob os tratamentos do $1^{\circ}$ cultivo $(0,150$, 300 e $450 \mathrm{~kg} / \mathrm{ha}$ de $\mathrm{K}_{2} \mathrm{O}$ ). Vale lembrar que o experimento foi implantado em um Cambissolo Háplico, com argila de atividade baixa e $\mathrm{CTC}=12,6 \mathrm{cmol}_{\mathrm{c}} / \mathrm{dm}^{3}$, ou seja, esses solos apresentam pequena capacidade de adsorver $\mathrm{K}$, o que pode ter levado a movimentação do nutriente aplicado no $1^{\circ}$ cultivo por lixiviação e menor aproveitamento pela planta em função do restrito sistema radicular da beterraba. $\mathrm{O}$ volume de precipitação desde a implantação do $1^{\circ}$ cultivo até a colheita do $2^{\circ}$ cultivo foi elevado, de $1181 \mathrm{~mm}$, contribuindo para a mobilidade vertical descendente do $\mathrm{K}$, que acontece principalmente por fluxo de massa, em decorrência das forças gravitacionais. Nesse processo, os fatores que mais influenciam positivamente a descida são as concentrações na solução do solo e o volume de água que percola, independentemente da condição; entretanto, a mobilidade no perfil estará dependente de uma boa precipitação pluvial no período (Ernani et al., 2007).

A maior exportação de $\mathrm{K}$ pela raiz ocorreu com $600 \mathrm{~kg} /$ ha de $\mathrm{P}$ aplicados no $1^{\circ}$ cultivo e $66 \%$ de reposição de $P$ com a planta exportando nas raízes 168,56 $\mathrm{kg} / \mathrm{ha}$ de $\mathrm{K}$. Para P na raiz, o melhor resultado foi obtido, também, com 600 $\mathrm{kg} /$ ha de $\mathrm{P}_{2} \mathrm{O}_{5}$ no $1^{\circ}$ cultivo e reposição de $66 \%$ de $\mathrm{P}$, tendo a planta exportado o equivalente a $26,64 \mathrm{~kg} / \mathrm{ha}$ de $\mathrm{P}$ do solo.

A CQFS-RS/SC (2004) recomenda para a adubação de reposição uma dose padronizada de $100 \mathrm{~kg} / \mathrm{ha}$ de $\mathrm{K}_{2} \mathrm{O}$ e $100 \mathrm{~kg} / \mathrm{ha}$ de $\mathrm{P}_{2} \mathrm{O}_{5}$, independente das características do solo ou produtividade obtida anteriormente. Para o Cambissolo Háplico da região do Alto Vale do Itajaí, com 2,5 mg/dm 3 de P no solo e teor de argila de $24 \%$, a proposta para a adubação de reposição é de 396 kg/ha de P e, para $\mathrm{K}$ uma adubação de reposição de $297 \mathrm{~kg} /$ ha de K, uma vez que o teor de $\mathrm{K}$ no solo é de $30 \mathrm{mg} / \mathrm{dm}^{3}$ e CTC 12,6 $\mathrm{cmol}_{\mathrm{c}} / \mathrm{dm}^{3}$.

No primeiro cultivo, a produção máxima de beterraba foi obtida com $379 \mathrm{~kg} / \mathrm{ha}$ de $\mathrm{P}_{2} \mathrm{O}_{5}$ e a exportação de $\mathrm{P}$ pela planta foi de $21,3 \mathrm{~kg} / \mathrm{ha}$, não sendo observada resposta à adubação potássica. Entretanto, a exportação do nutriente pelas plantas foi elevada.

No segundo cultivo, a máxima produtividade obtida com as maiores doses aplicadas no primeiro cultivo $(600 \mathrm{~kg} /$ ha de $\mathrm{P}_{2} \mathrm{O}_{5}$ e $450 \mathrm{~kg} / \mathrm{ha}$ de $\mathrm{K}_{2} \mathrm{O}$ ) e $66 \%$ a dose de reposição com exportação de $16,88 \mathrm{~kg} / \mathrm{ha}$ de $\mathrm{P}$ pela folha e $26,64 \mathrm{~kg} /$ ha de P pela raiz e $100,24 \mathrm{~kg} / \mathrm{ha}$ de $\mathrm{K}$ pela folha e $168,56 \mathrm{~kg} /$ ha de $\mathrm{K}$ pela raiz.

\section{REFERENNCIAS}

ALMEIDA, JA; KAMPF, N; ALMEIDA, R. 1997. Caracterização mineralógica de solos originados de rocha pelítica nos patamares do Alto Rio Itajaí e no Planalto de Lages (SC). Revista Brasileira de Ciência do Solo 21: 181-190.

ALVES, AU; PRADO, RM; GONDIM, ARO; FONSECA, IM; CECÍLIO FILHO, AB. 2008. Desenvolvimento e estado nutricional da beterraba em função da omissão de nutrientes. Horticultura Brasileira 26: 292-295.

AVALHAES, CC; PRADO, RM; GONDIM, ARO; ALVES, AU; CORREIA, MAR. 2009. Rendimento e crescimento da beterraba em função da adubação com fósforo. Scientia Agrária 10: 75-80.

CAMPO, J; MAASS, MJ; JARAMILLO, VJ; YRÍZAR, AM. 2000. Calcium, potassium, and magnesium cycling in a Mexican tropical dry forest ecosystem. Biogeochemistry 49: 21-36.

CEASA-SC. 2007. Estatística dos produtos comercializados. Disponível em: http:// cepa.epagri.sc.gov.br/Ceasa/Boletim $\% 20$ CEASA\%20Junho.pdf

CHITARRA, MIF; CHITARRA, AB. 2005. Pós-colheita de frutas e hortaliças: fisiologia e manuseio. Lavras: Fundação de apoio ao Ensino, Pesquisa e Extensão, 785 p.

COMISSÃO DE QUÍMICA E FERTILIDADE DO SOLO CQFS-RS/SC. 2004. Manual de adubação e calagem para os Estados do Rio Grande do Sul e Santa Catarina. Porto Alegre: UFRGS. 400p.

DUARTE, TS; HILLESHEIM, PC; SCHEIDT, BT. 2012. Estudo comparativo de genótipos de beterraba cultivados na primavera/verão no Alto Vale do Itajaí, SC. Horticultura Brasileira 30: 2957-2963.

EPAGRI - Estação Experimental da Epagri - Ituporanga-SC. 2010. Banco de dados: Informação pessoal.
ERNANI, PR; BAYER, C; ALMEIDA, JA; CASSOL, PC. 2007. Mobilidade vertical de cátions influenciada pelo método de aplicação de cloreto de potássio em solos com carga variável. Revista Brasileira de Ciência do Solo 31: 393-402.

FILGUEIRA, FAR. 2012. Novo manual de olericultura: agrotecnologia moderna na produção e comercialização de hortaliças. $3^{\mathrm{a}}$ edição. Viçosa: UFV. 418p.

GRANGEIRO, LC; NEGREIROS, MZ; SOUZA, BS; AZEVEDO, PE; OLIVEIRA, SL; MEDEIROS, MA. 2007. Acúmulo e exportação de nutrientes em beterraba. Ciência e Agrotecnologia 31: 267-273.

HAAG, HP; MINAMI, K. 1987. Nutrição mineral de hortaliças: LXIII. Requerimento de nutrientes pela cultura da beterraba. Anais da Escola Superior de Agricultura Luiz de Queiroz, Piracicaba, v. 44, p. 401-407.

KAMINSKI, J; MOTERLE, DF; RHEINHEIMER, DS; GATIBONI, LC; BRUNETTO, G. 2010. Potassium availability in a hapludalf soil under long term fertilization. Revista Brasileira de Ciência do Solo 34: 783-791.

KHATOUNIAN, CA. 2001. A reconstrução ecológica da agricultura. Botucatu: Agroecologica. 348 p.

MAGRO, FO. 2012. Efeito do composto orgânico e adubação potássica em atributos do solo $e$ da beterraba. Botucatu: UNESP. 109p. (Tese doutorado).

MARINKOVIĆ, B; CRNOBARAC, J; JAĆIMOVIĆ, G; RAJIĆ, M; LATKOVIĆ, D; AĆIN, V. 2010. Sugar yield and technological quality of sugar beet at different levels of nitrogen fertilization. Research Journal of Agricultural Science 42.

NOVAIS, RF; SMYTH, TJ. 1999. Fósforo em solo e planta em condições tropicais. Viçosa: UFV. 399p.

RAIJ, B; CANTARELLA, H; QUAGGIO, IA; FURLANI, AMC. 1997. Recomendações de adubação e calagem para o Estado de São Paulo. 2.ed. Campinas: IAC, 285p. (Boletim Técnico, 100).

RESENDE, GM; CORDEIRO, GG. 2007. Uso da água salina e condicionador de solo na produtividade de beterraba e cenoura no semiárido do submédio São Francisco. Petrolina: Embrapa Semi-Árido. Comunicado Técnico, 128. $4 \mathrm{p}$.

SAS Institute Inc ${ }^{\circledR}$. 2003. SAS Ver. 9.1. 3 Cary. SEDIYAMA, M; SANTOS, MR; VIDIGAL, SM; SALGADO, LT. 2011. Produtividade e exportação de nutrientes em beterraba cultivada com cobertura morta e adubação orgânica. Revista Brasileira de Engenharia Agrícola e Ambiental 15: 883- 889.

TEDESCO, MJ. 1995. Análises de solo, plantas e outros materiais. Porto Alegre: UFRGS. (Boletim Técnico de Solos, 5). 174p.

TURK, M. 2010. Effects of fertilization on root yield and quality of fodder beet (Beta vulgaris var. crassa Mansf.) Bulgarian Journal of Agricultural Science 16: 212-219 